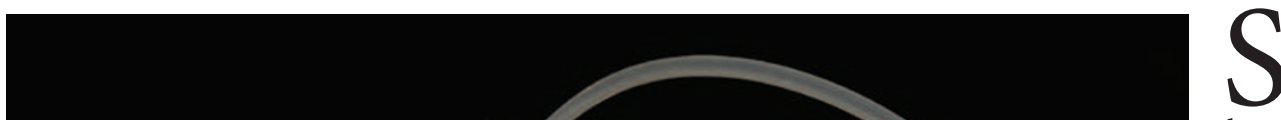

S arah Gilpin is talking to a pair of opalescent human lungs. "You guys are so cool," she says, peering into a keg-sized glass bioreactor at Massachusetts General Hospital (MGH) in Boston. The lungs float inside, puffing up as 40 litres of soapy fluid is pumped through their network of blood vessels. Less than a week earlier, these lungs belonged to a 47-year-old New Yorker, allowing him to take one last breath before dying of cardiac arrest. Now, his donated lungs have been stripped of their cells and genetic material. All that remains are two cone-shaped objects made of collagen and other structural proteins, dangling as though in suspended animation.

Gilpin, a postdoctoral fellow, is using such protein scaffolds to try to grow a set of bioartificial lungs in the laboratory. Together with Harald Ott, a cardiothoracic surgeon at MGH, Gilpin is devising a protocol to flush out the cells from donated lungs that are ineligible for direct transplantation. Eventually, they plan to seed the support structure with stem cells and to direct these cells to develop, or differentiate, into the various cell types in the lung, thereby rebuilding functioning tissue. Using the techniques the lab has developed so far, Ott has already engineered bioartificial rat lungs that can perform the appropriate gas-exchange functions when transplanted back into living rats ${ }^{1}$. Now, he and Gilpin are scaling up to humans.

Success in their quest would bring good news to the estimated 65 million people worldwide who live with chronic obstructive pulmonary disease (COPD), a lung disorder mainly caused by exposure to tobacco smoke and airborne pollutants. Medication can often help treat the symptoms and complications of COPD, such as breathlessness and chronic coughing. But, for many people with severe forms of the disease, the only option is to replace a bad set of lungs with a good set. Relatively few lungs are donated, however, and most of these are damaged and therefore unsuitable for transplantation (see 'Organ refit'). So, each year, around the world, only a few thousand patients with COPD receive a life-saving transplant. New sources of healthy lungs or devices that serve the same purpose are desperately needed.

Such technologies are lacking. "Count the number of devices you can use if your heart is failing, and you can make a nice long list. But if your lung is failing, you have very few options," says Scott Merz, president and co-founder of Michigan Critical Care Consultants in Ann Arbor, a company that designs and develops 'lung-support' technologies.

Lab-grown lungs, reconstructed from damaged donor lungs and seeded with a patient's own cells, offer one attractive option. What's more, these bioartificial transplants could have a major advantage over their natural counterparts: by
D NATURE.COM Artificial pancreas to make diabetes more manageable: go.nature.com/spn6zs

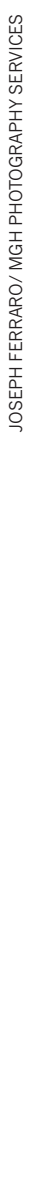


incorporating a patient's own (autologous) cells, the lungs would be less prone to rejection by the immune system than lungs transplanted directly from a donor, and recipients wouldn't have to take lifelong cocktails of immunosuppressive agents. However, considering that Gilpin, on this particular July morning, was only part way through removing the cells from her second set of human lungs and that no other team has reported success with this approach on a similar scale, the research is still a long way from the clinic.

"We're a couple of decades away from having an autologous, tissue-engineered lung," says Laura Niklason, a biomedical engineer at Yale University in New Haven, Connecticut, who has independently created bioartificial rodent lungs ${ }^{2}$. "There are technological hurdles, and there are stem-cell differentiation hurdles. But I don't think anything is insurmountable."

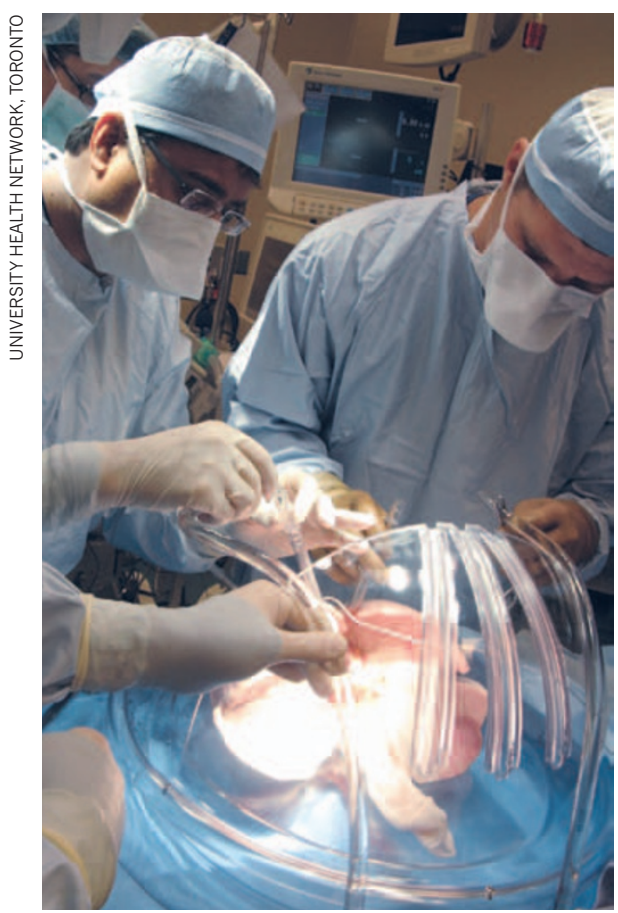

Surgeons strip lungs of their original cells.

In the meantime, many academic and industry researchers are turning to mechanical devices that can help the lungs to carry out their main function: gas exchange, specifically the removal of carbon dioxide from the bloodstream and the delivery of oxygen. The first such products are already on the market, and the next generation of 'artificial lungs' is not far behind.

\section{OUTSIDE THE BOX}

Novalung, a device manufacturer based in Talheim, Germany and spun off from Merz's company, is the market leader in lung-support technologies. The company's interventional lung assist (iLA) membrane ventilator takes blood from a patient's thigh and circulates it under ambient pressure from the femoral artery through a rod lined with hundreds of tubes full of pure oxygen. Oxygenated blood then flows back into the femoral vein. This system, which was approved in Europe and a handful of other countries in the past ten years for providing respiratory support for up to 29 days, has mainly been used as a stopgap for people urgently awaiting lung transplants. But doctors are beginning to test whether this device, and similar technologies, can be used earlier in the treatment process to avoid the need for transplants altogether.

Long before needing a new set of lungs, a patient with COPD will be well acquainted with the hospital emergency department because of frequent episodes of severe breathing difficulty. These attacks - called acute exacerbations occur, on average, two to three times a year in people with moderate-to-severe COPD. To give the lungs a break and help the patient breathe during one of these episodes, doctors usually attempt to deliver air through a face mask. Although these masks are easier to apply and less cumbersome for patients to wear than the iron lungs of the early twentieth century, maskbased ventilation doesn't work for everybody. For example, individuals who are not fully conscious and cooperative are not considered good candidates. So, if the mask approach fails, doctors turn to more invasive techniques, in which a breathing tube is inserted into the windpipe and a ventilating machine pushes air directly into the lungs. Such mechanical ventilation can save lives during a COPD attack. But it also significantly increases the risk of pneumonia, and forcing the air into the lungs can cause injury.

Lung support devices such as the iLA might obviate the need for invasive ventilation during exacerbations. For example, in a pilot study of the iLA system reported in June 2012 at the annual meeting of the German Society for Internal Intensive and Emergency Medicine in Cologne, doctors at the University Medical Center Hamburg-Eppendorf in Germany helped 13 of 14 patients with COPD avoid tracheal intubation.

"You have something that breathes outside the patient, allowing the lungs to recover and heal rather than being forced to perform by mechanical ventilation," says Georg Matheis, a managing director of Novalung, based in Heilbronn, Germany. "This might affect the long-term cause of COPD by mitigating or avoiding the damage inflicted by these exacerbations." In this way, patients who are protected against progressive damage by a system such as Novalung's might ultimately live longer or have an easier time breathing.

Novalung is now moving towards a new iLA design, with a pump that exchanges blood through the jugular veins, in the neck. This approach gives patients greater mobility, as well as lowers the risk of heart attack and other problems associated with an arterial puncture. But Novalung is not alone in pursuing this strategy: another company, ALung

\section{ORGAN REFIT \\ All donations accepted}

More than $80 \%$ of donated lungs worldwide are too damaged to transplant. These lungs can still be used to study tissueengineering techniques, such as those used for generating bioartificial lungs in the laboratory of Harald Ott, a cardiothoracic surgeon at Massachusetts General Hospital in Boston. But, with many people dying while on the waiting list for a lung transplant, researchers are beginning to explore whether the unsuitable lungs might be of immediate benefit to patients, especially if some of the tissue damage can be repaired.

In a 2012 study, a British team of doctors assessed the survival of nearly 1,300 lungtransplant recipients, some 500 of whom had received lungs from donors who smoked ${ }^{5}$, which is permitted under UK policy. As might be expected, transplant recipients whose lung donors had not smoked survived longer. But recipients of smokers' lungs fared better than patients with COPD who remained on the waiting list. "It's better to get a lung with a smoking history than no lung at all," says Lorraine Ware, a doctor who studies lung transplantation at the Vanderbilt University School of Medicine in Nashville, Tennessee.

However, before doctors race to transplant suboptimal lungs from smokers, they might consider applying a recently developed technique called the Toronto XVIVO Lung Perfusion System. Developed by Shaf Keshavjee and his colleagues at the University Health Network in Toronto, Canada, the procedure involves pumping a protein and nutrient solution into the donor lungs and ventilating them with an oxygen gas mixture for several hours to repair damage caused by swelling or inflammation.

In a 2011 report, the Toronto team showed that only $15 \%$ of suboptimal lungs prepared in this way showed signs of acute organ injury three days after transplantation, in contrast to $30 \%$ of the supposedly healthy lungs that were transplanted without such preparation ${ }^{6}$. “The advantage isn't just that we can use more lungs but that we also make these transplants safer and more predictable," says Keshavjee, adding that around $20 \%$ of organs transplanted at the Toronto Lung Transplant Program undergo this perfusion treatment. (The Toronto program, like those in the UK, allows the use of smokers' lungs, which Keshavjee says make up 30-40\% of lungs considered for transplant.)

"This is our first foray into partially engineering or repairing donor lungs," Keshavjee notes. If he succeeds more broadly, maybe bioartificial lungs won't be necessary after all. E.D. 
Technologies based in Pittsburgh, Pennsylvania, is advancing a similar device as an alternative to mechanical ventilation.

In June 2012, at the 58th Annual Conference of the American Society of Artificial Internal Organs in San Francisco, California, ALung reported success with its Hemolung device, showing that patients with severe exacerbations of COPD experienced, on average, $28 \%$ reductions in their blood levels of carbon dioxide within 24 hours of the start of Hemolung treatment. "We're bringing the technology down to a level of safety, simplicity and superior efficacy, all with a lower level of invasiveness," says Scott Morley, a biomedical engineer who heads product management at ALung. The company plans to file for European approval of the Hemolung system later this year.

\section{POOR EXCHANGE}

Not everyone is convinced that lung-support devices offer any real advantage over mechanical ventilation for treating COPD. "Right now, the risk-benefit ratio of those two is very close, and it's hard to tell how that's going to play out," says Joseph Zwischenberger, a cardiothoracic critical-care doctor at the University of Kentucky in Lexington, who has been working on artificial lungs for about 30 years. "There are those who think the technology has evolved to the point where the risk of the devices is less than the risk of being on a ventilator, and then there are a large majority who say, 'Nope, it's just not there yet."

One of the biggest problems with the existing technologies is their tendency to clot: rapid blood cells encounter something akin to a traffic jam as they flow into and out of the devices. The devices are also bulky, and only limited gas exchange happens as the blood passes over the oxygen-filled fibres. Thus, many advocates of lung-support systems are looking to completely redesign the technology and are turning to the science of very small volumes - microfluidics.

Using microfluidics "enables you to get much more surface area for gas exchange per unit blood volume than the traditional devices," says William Federspiel, a bioengineer at the University of Pittsburgh and a co-founder of ALung. "That could allow for a more compact device that is potentially efficient enough to run off room air," unlike existing devices, which typically require a tank of pure oxygen.

Some success has been achieved already. Air delivered at near ambient pressures was able to oxygenate blood in a microfluidic device designed by Joseph Potkay and Only a pair of healthy human lungs can reverse the downward progression

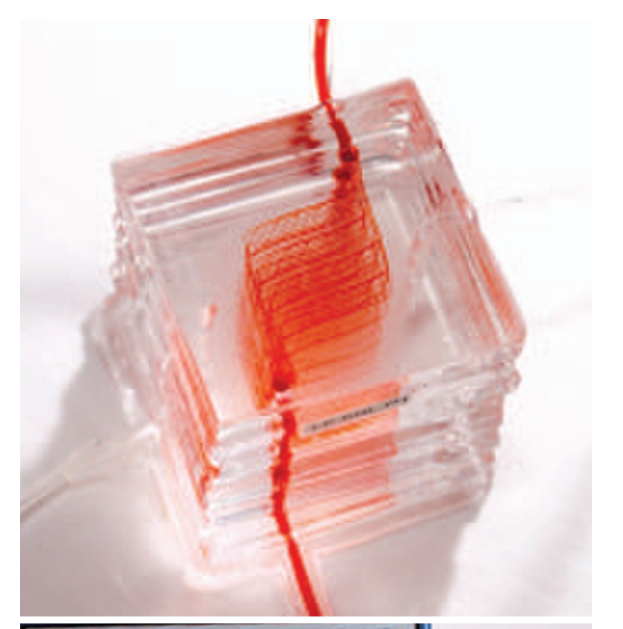

devices on the same size scale as the structures in the natural lung."

\section{SIZE MATTERS}

The device still has some kinks that need to be worked out. For one thing, it would take more than 1,000 of them on top of one another to provide just one-quarter of the body's baseline gasexchange support. And Potkay's team designed the device to maximize gas exchange but didn't optimize it to prevent clotting. By more closely mimicking natural blood-vessel architecture, however, a few research teams have designed microfluidic chips that circumvent the clotting problem, paving the way for long-term lungsupport devices that could aid even mobile patients with COPD.

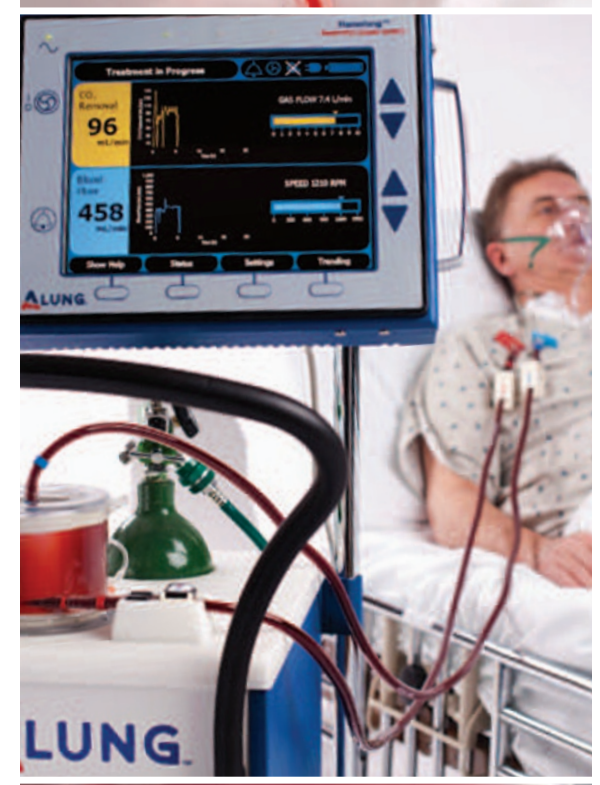

Joseph Vacanti, a transplant surgeon at $\mathrm{MGH}$, is leading one such effort. Vacanti helped Ott to engineer bioartificial rat lungs, but he is now focused on developing a microfluidic device. Vacanti's group has built a device with a bifurcating architecture that closely matches the branching of blood vessels in the body ${ }^{4}$. With an eye to miniaturization, his group is now working to make the system out of collagen instead of silicone, a material that introduces much dead space into the device. "We've got a lot of work to do on choosing the best material and making it in the thinnest possible way so the size of the thing is either wearable or implantable," says Vacanti.

Across the river from MGH, Jeffrey Borenstein, director of the Biomedical Engineering Center at the Charles Stark Draper Laboratory in Cambridge, who has his own microfluidic lung prototype, is focused on optimizing another part of the design. To reduce the possibility of clotting, Borenstein and his colleagues are seeding the channels of their branching gas-exchange device with the cells that line blood vessels endothelial cells - and, so far, they have seen no decline in the rates of gas transfer. "As we think about clinical implementation we really want to make this as natural a device as possible so we're not always dealing with these blood interactions with synthetic surfaces," Borenstein says.

Gilpin, the MGH postdoc, praises the efforts to develop lung-support devices and hopes they will soon provide some relief for people with COPD. But, ultimately, she says, only a pair of healthy human lungs can reverse the downward progression of this devastating condition. She leans her elbows on the lab bench, props her face in her hands, and gazes at the stripped set of lungs. "Seeing this makes me think we have the platform," she says. "You'd never be able to create this. Only nature can create this."

Potkay and his team created a credit-card-sized device with artificial capillaries that achieved gas-exchange rates far surpassing those of current devices, without the need for pure oxygen. "We're pushing the size limits of this technology," says Potkay, who is now at the Veterans Affairs Medical Center in Ann Arbor. But "through [such] microfabrication, we can get

\section{Elie Dolgin is a news editor at Nature Medicine.}

\footnotetext{
1. Ott, H. C. et al. Nature Med. 16, 927-933 (2010).

2. Petersen, T. H. et al. Science 329, 538-541 (2010).

3. Potkay, J. A. et al. Lab Chip 11, 2901-2909 (2011).

4. Hoganson, D. M. et al. Lab Chip 11, 700-707 (2011).

5. Bonser, R. F. et al. Lancet 380, 747-755 (2012)

6. Cypel, M. etal. N. Engl. J. Med. 364, 1431-1440 (2011).
} 